\title{
$\ln _{0.5} \mathrm{Ga}_{0.5}$ As quantum dot intermixing and evaporation in GaAs capping layer growth
}

\author{
Jeong-Sik Lee, ${ }^{\text {a) }}$ Hong-Wen Ren, Shigeo Sugou, and Yasuaki Masumoto \\ Single Quantum Dot Project, ERATO, JST, Tsukuba Research Consortium, 5-9-9 Tokodai, \\ Tsukuba 300-2635, Japan
}

(Received 26 June 1998; accepted for publication 14 September 1998)

\begin{abstract}
Our study of GaAs growth over self-assembled $\mathrm{In}_{0.5} \mathrm{Ga}_{0.5}$ As quantum dots grown by metalorganic vapor-phase epitaxy showed that GaAs capping layer surface morphology at the onset strongly depended on temperature. Incompletely capped $\mathrm{In}_{0.5} \mathrm{Ga}_{0.5} \mathrm{As}$ islands were elongated toward [110], indicating anisotropy in intermixing. During higher-temperature growth interruption, islands show craters in quantum dot centers. Craters become hexagonal holes whose depth matches GaAs capping layer thickness. Postannealing photoluminescence spectra show no peak corresponding to overly large quantum dot radiation, indicating that growth interruption after capping layer formation at a certain thickness eliminates overly large quantum dots. (C) 1998 American Institute of Physics.

[S0021-8979(98)00924-4]
\end{abstract}

\section{INTRODUCTION}

Self-assembled islands formed in Stranski-Krastanow growth mode have attracted attention for use as quantum dot (QD) structures because potential ex situ pixellation damage and contamination are avoided. ${ }^{1,2}$ Despite extensive study on self-assembled QD growth and optical characterization, problems such as nonuniform size distribution and uncertain QD height remain, ${ }^{3}$ leading to discrepancies between observed quantization effects and theory. QD height fluctuation broadens photoluminescence (PL) peaks. Structural disorder such as intermixing during overlayer growth further complicates matters. ${ }^{4}$ Intermixing and evaporation change QD size, shape, and composition, resulting in discrepancies between expected and actual structure. Despite the importance of well-defined quantum structure fabrication, a few studies have focused on intermixing and evaporation during capping layer growth following QD formation.

This article details the intermixing and evaporation involving metalorganic vapor-phase epitaxy (MOVPE) used to grow $\mathrm{In}_{0.5} \mathrm{Ga}_{0.5}$ As QDs during GaAs capping layer growth, focused on QD structure dependence on growth temperature $\left(T_{\mathrm{g}}\right)$ and capping layer thickness. Results show selective evaporation of exclusively high QDs, whose height exceeds the GaAs capping layer thickness. These results suggest that, to eliminate such undesirable QDs, the application of growth interruption following capping layer growth to a certain thickness would be feasible.

\section{EXPERIMENTS}

Our growth system is based on a conventional MOVPE system, Emcore Discovery 75 . The working pressure was 80 $\mathrm{hPa}$. Using $1400 \mathrm{rpm}$ substrate rotation throughout the growth ensured the source gas flow. All the $\mathrm{In}_{0.5} \mathrm{Ga}_{0.5} \mathrm{As}$ QD samples were grown on GaAs (001) substrates at $480^{\circ} \mathrm{C}$.

${ }^{a)}$ Corresponding author; electronic mail: jslee@obl.cl.nec.co.jp
Islands were capped by GaAs layer with or without interrupting growth at various $T_{\mathrm{g}}$. Triethylgallium (TEG), trimethylindium (TMI), and arsine $\left(\mathrm{AsH}_{3}\right)$ were used as source materials. Flow rates of TEG and $\mathrm{AsH}_{3}$ for $\mathrm{GaAs}$ were $6.65 \times 10^{-6}$ and $1.83 \times 10^{-3} \mathrm{~mol} / \mathrm{s}$, and TEG, TMI, and $\mathrm{AsH}_{3}$ for $\mathrm{In}_{0.5} \mathrm{Ga}_{0.5} \mathrm{As}$ were $1.33 \times 10^{-6}, 1.19 \times 10^{-6}$, and $1.83 \times 10^{-3} \mathrm{~mol} / \mathrm{s}$, respectively. The GaAs growth rate was 0.5 monolayer (ML)/s and that of $\operatorname{In}_{0.5} \mathrm{Ga}_{0.5}$ As was $0.2 \mathrm{ML} / \mathrm{s}$.

Surface morphologies were studied by atomic force microscopy (AFM) in air. Room temperature (RT) PL spectra was measured under excitation by an $\mathrm{Ar}^{+}$laser tuned to $488 \mathrm{~nm}$.

\section{RESULTS AND DISCUSSIONS}

We began by studying intermixing dependence on QD size growing samples with large QD size distribution intentionally [Fig. 1(a)]. The QD size distribution in Fig. 1(a) is displayed in Fig. 2, showing a bimodal distribution. Similar bimodal QD size distribution has also been reported on InAs QDs grown on (001) substrates. ${ }^{5}$ The average height and diameter of smaller QDs are 80 and $320 \AA$. Larger QDs are about $110 \AA$ in height and $400 \AA$ in diameter, respectively. A GaAs overlayer was grown at the different $T_{\mathrm{g}}$ on the QD structure. Figure 1(b) shows the AFM image of the sample covered with a 60 - $\AA$-thick GaAs capping layer at the same $T_{\mathrm{g}}$ of $480{ }^{\circ} \mathrm{C}$. Island density in uncapped [Fig. 1(a)] and capped samples was similar, indicating that $\mathrm{In}_{0.5} \mathrm{Ga}_{0.5}$ As QDs originated the islands. Islands are elongated toward the [110] azimuth, being two times larger in the [110] direction. Moreover, the islands have craters at their centers that clearly become holes in larger QDs. Craters and holes make islands lower than as-grown QDs. All of these features indicate intermixing at the interface between the $\mathrm{In}_{0.5} \mathrm{Ga}_{0.5} \mathrm{As}$ QD and GaAs capping layer. Intermixing is assumed to be enhanced at larger QDs because of the higher In composition at the 

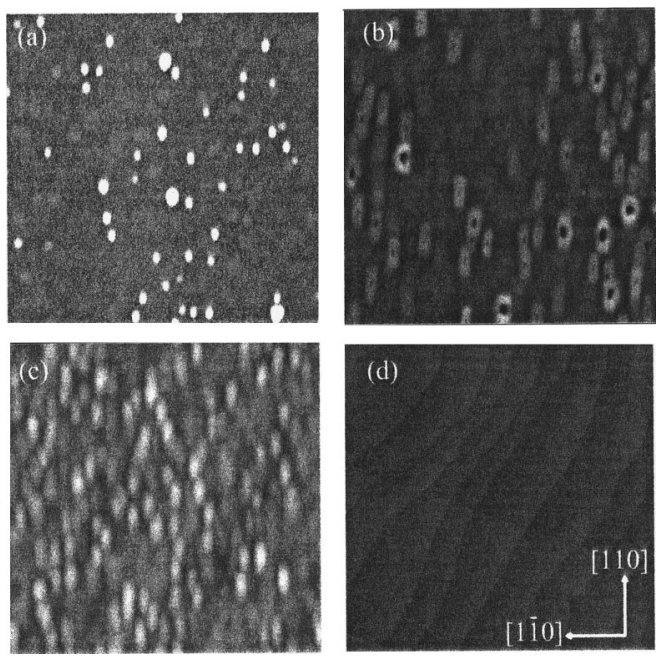

FIG. 1. AFM images of (a) as-grown $\operatorname{In}_{0.5} \mathrm{Ga}_{0.5} \mathrm{As}$ QDs and (b) samples covered with a $60 \AA$ GaAs capping layer grown at $480^{\circ} \mathrm{C}$. (c) At lower $T_{\mathrm{g}}$ $\left(450^{\circ} \mathrm{C}\right)$, no depression occurs in island centers and (d) a smooth surface is obtained after the 150- $\AA$-thick GaAs capping layer growth.

interface due to segregation or larger dislocation density, hence, the holes. The above features also suggest the occurrence of evaporation, discussed later.

Craters and holes did not form when the GaAs capping layer was grown at lower $T_{\mathrm{g}}\left(450^{\circ} \mathrm{C}\right)$ as shown in Fig. 1(c), and QDs were about the same height despite increased island shape diameter and anisotropy. Island size anisotropy appears to be related to anisotropy in intermixing and in migration length of $\mathrm{Ga}$ precursors. This is distinguished easily because island height decreases simultaneously with intermixing. ${ }^{4}$ Thus, the anisotropy in diameter is due mainly to the anisotropy of overlayer growth rate, as suggested by similar island height. Island elongation toward [110] agrees well with anisotropy in the migration length of Ga precursors in MOVPE growth. ${ }^{6}$ A flat surface is attained when QD embedding completed as seen in Fig. 1(d).

Unique phenomena also occur after growth interruption at higher temperature, i.e., annealing, after growing the GaAs capping layer thinner than QDs height. Figure 3(a) shows the

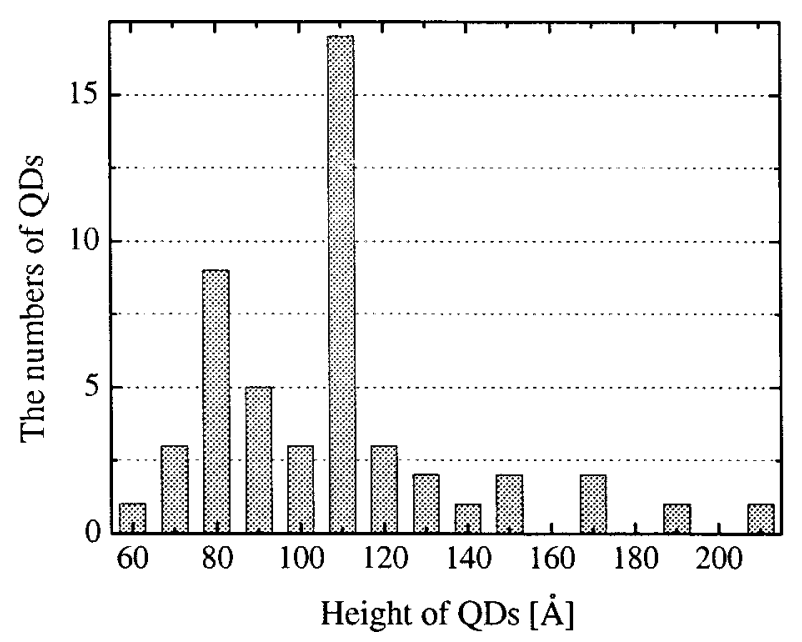

FIG. 2. QD height histogram obtained from Fig. 1(a). (a)

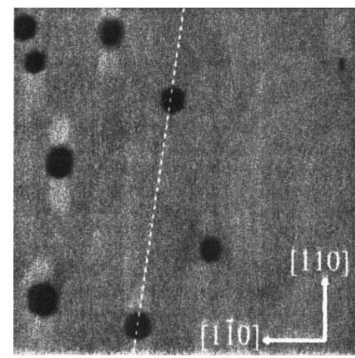

[nm]

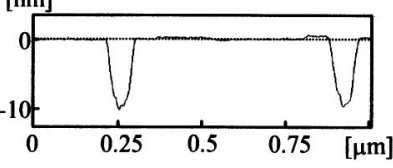

(b)

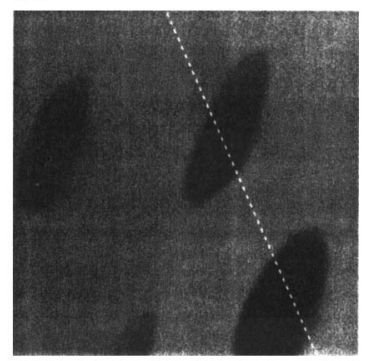

[nm]

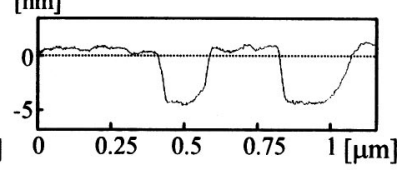

FIG. 3. AFM images of samples after annealing at $640{ }^{\circ} \mathrm{C}$ following (a) 100 $\AA$ and (b) $50 \AA$ GaAs capping layer growth at $450{ }^{\circ} \mathrm{C}$. The dotted line across the AFM image shows the path taken for the profile, below. AFM profiles appear below.

surface AFM image of the annealed QD sample with $100-\AA$ thick GaAs capping layer grown at $450{ }^{\circ} \mathrm{C}$. The annealing temperature and time are $640{ }^{\circ} \mathrm{C}$ and $1 \mathrm{~min}$, respectively. The AFM image presents surface morphology, which consists of flat regions and holes. In the sample surface immediately after capping layer growth (not shown), the holes cannot be seen but mound-like islands occur in the place of holes. QDs higher than $100 \AA$ in Fig. 1(a) and holes show similar density. The AFM profile also shows that hole depths are consistent with capping layer thickness. Thus, holes originate in QDs higher than the capping layer. Evaporation following atom intermixing and redistribution with migration at the top of the exposed QD cause holes to form. Mounds on both sides (in the [110] direction) of holes present rough images of QD structure intermixing and evaporation as is shown in Fig. 2(a). These mounds are assumed to consist of atoms composing QDs. During annealing, QD region atoms creep and migrate on the surface. Although most atoms eventually evaporate, they leave their trace behind in the form of mounds. These atoms are strongly directed toward the [110] azimuth, coinciding with island shape anisotropy caused by anisotropy in Ga precursor migration length.

Holes twice the size of as-grown QDs evidence considerable intermixing in embedded region due to high annealing temperature, i.e., intermixing occurs even in fully embedded QDs, broadening the PL peak. The clearly hexagonal hole shape indicates facet formation. The AFM profile for different directions indicates that hole sidewalls consist of $\{111\}$ and $\{110\}$ facets. Why facets form is unclear, but facets resemble those in mesa etching. ${ }^{7}$ Stable states appear to exist at low Miller index planes that resist intermixing or reevaporation. The participation of dislocation may also be a factor.

When the same process is conducted for samples with a thinner capping layer, large holes occur on the surface as seen in Fig. 3(b). The increased number of incompletely capped $\mathrm{In}_{0.5} \mathrm{Ga}_{0.5}$ As islands increases hole density. As holes coalesce, large holes form in thinner capping layer. The flat bottoms of holes whose depths match capping layer thick- 


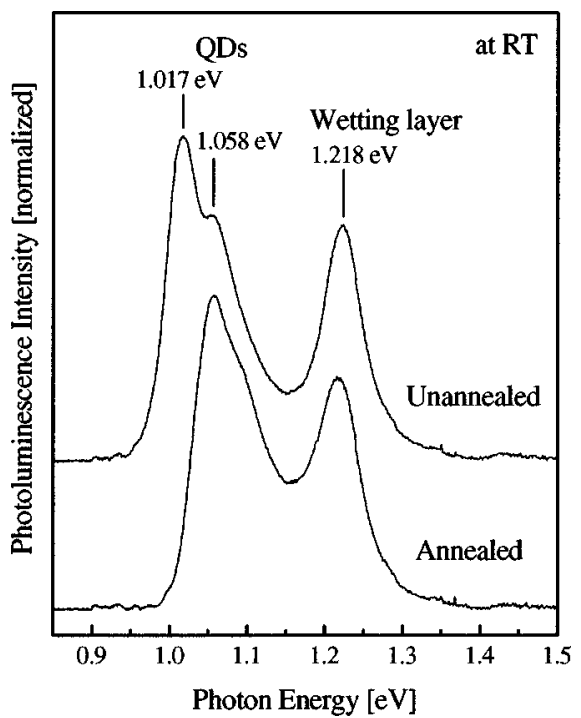

FIG. 4. RT PL spectra of annealed and unannealed $\operatorname{In}_{0.5} \mathrm{Ga}_{0.5} \mathrm{As}$ QD samples after the $100-\AA$-thick GaAs capping layer growth at $450{ }^{\circ} \mathrm{C}$.

ness further support the idea of hole formation following intermixing and evaporation. Thus, annealing after capping layer growth at a specified thickness would eliminate overly large QDs.

Figure 4 shows the RT PL spectra of annealed and unannealed $\operatorname{In}_{0.5} \mathrm{Ga}_{0.5}$ As QD samples following $100 \AA$ GaAs capping layer growth at $450{ }^{\circ} \mathrm{C}$. For the annealed sample, $T_{\mathrm{g}}$ was set to conventional GaAs growth of $640^{\circ} \mathrm{C}$ and a 300$\AA$-thick GaAs overlayer was grown. For the unannealed sample, a 150 - $\AA$-thick GaAs capping layer was grown at $450{ }^{\circ} \mathrm{C}$ before $T_{\mathrm{g}}$ was set to $640{ }^{\circ} \mathrm{C}$ to grow the 200 - $\AA$-thick GaAs layer. Three peaks occur for the unannealed sample. The peak at $1.218 \mathrm{eV}$ is for a wetting layer. The relatively large linewidth in the wetting layer peak is due to the large fluctuation in layer thickness. From the result of excitation power dependent PL measurement, the peaks at 1.017 and $1.058 \mathrm{eV}$ were confirmed to be the radiation from the QDs with bimodal size distribution as was shown in Fig. 2. Since the peak at $1.058 \mathrm{eV}$ was clearly distinguishable under low excitation power of $0.1 \mathrm{~W} / \mathrm{cm}^{-2}$, the peak does not originate from higher energy subband of the peak at $1.017 \mathrm{eV}$ but originates from the ground state of smaller QDs (data not shown). Similar PL result reflecting bimodal QD size distri- bution has been reported by $\mathrm{Yu}$ et al..$^{5}$ In the PL spectra of annealed sample, the peak at $1.017 \mathrm{eV}$ disappeared whereas no change was observed in the peak at 1.058 and $1.218 \mathrm{eV}$. The peaks at 1.058 and $1.218 \mathrm{eV}$ originate from the fully embedded QDs and wetting layer by the GaAs capping layer before annealing. The structures keep their shape during the annealing because they are protected by the GaAs capping layer. Consequently, no difference was seen in the PL spectra of annealed and unannealed samples. On the other hand, the peak at $1.017 \mathrm{eV}$ is considered to be the radiation from the larger QDs (110 $\AA$ in height). Since the larger QDs are taller than the 100- $\AA$-thick GaAs capping layer, top of the QD structures were exposed on the surface. Therefore, evaporation of the larger QDs was enhanced during annealing and the QDs eliminate as was confirmed in Fig. 3(a). It is the reason why the PL peak at $1.017 \mathrm{eV}$ disappears after annealing. These results indicate that size uniformity can be improved by selectively evaporating overly large QDs using a certain GaAs capping layer thickness and $T_{\mathrm{g}}$.

\section{CONCLUSIONS}

Intermixing and evaporation during GaAs capping layer growth over MOVPE grown self-assembled $\mathrm{In}_{0.5} \mathrm{Ga}_{0.5} \mathrm{As}$ QDs were studied. $\mathrm{In}_{0.5} \mathrm{Ga}_{0.5}$ As islands partially capped by GaAs layer showed "craters" at QD centers at higher GaAs growth temperature. Craters become clearly hexagonal holes whose depths corresponded to GaAs capping layer thickness after annealing indicating that QDs higher than the capping layer were evaporated by annealing. Postannealing PL spectra show no peak corresponding to overly large QD radiation, indicating that growth interruption after capping layer formation at a certain thickness eliminates overly large QDs. The results indicate that the annealing technique can be used to select QD size distribution.

${ }^{1}$ H. Sakaki, Jpn. J. Appl. Phys., Part 2 19, L735 (1980).

${ }^{2}$ L. Goldstein, F. Glas, J. Y. Marzin, M. N. Charasse, and G. L. Roux, Appl. Phys. Lett. 47, 1099 (1985).

${ }^{3}$ J.-Y. Marzin and G. Bastard, Solid State Commun. 92, 437 (1994).

${ }^{4}$ J. M. Garcia, G. Medeiros-Ribeiro, K. Schmidt, T. Ngo, J. L. Feng, A. Lorke, J. Kotthaus, and P. M. Petroff, Appl. Phys. Lett. 71, 2014 (1997).

${ }^{5}$ H. Yu, S. Lycett, C. Roberts, and R. Murray, Appl. Phys. Lett. 69, 4087 (1996).

${ }^{6}$ M. Kasu and T. Fukui, Jpn. J. Appl. Phys., Part 2 31, L864 (1992).

${ }^{7}$ S. Iida and H. Ito, J. Electrochem. Soc. 118, 768 (1971). 